\title{
SUBNORMAL WEIGHTED SHIFTS AND ASYMPTOTIC PROPERTIES OF NORMAL OPERATORS
}

\author{
J. J. BASTIAN AND K. J. HARRISON
}

\begin{abstract}
By Fuglede's theorem, an operator which commutes with a normal operator $N$ must also commute with all spectral projections of $N$. We show that only in special cases is the theorem true when "near-commutativity" replaces "commutativity". We show too that the restriction of a normal operator to an almost reducing subspace may be far from all normal operators on the subspace.
\end{abstract}

Introduction. One of the formulations of the Fuglede theorem is that if $N$ is a normal operator on a Hilbert space and $A$ is an operator such that $N A=A N$, then $E(\mathscr{S}) A=A E(\mathscr{S})$ where $E$ is the unique spectral measure associated with $N$ and $\mathscr{S}$ is an arbitrary Borel subset of the plane. It is not unreasonable to conjecture an asymptotic generalization of this fact. That is, if $N$ is a normal operator, $\mathscr{S}$ a Borel subset of the plane, and $A$ an operator such that $\|N A-A N\|$ is small, then $\|A E(\mathscr{S})-E(\mathscr{S}) A\|$ is small. Precisely, for every positive number $\varepsilon$, there exists a positive number $\delta$ such that $\|N A-A N\|<\delta$ implies $\|E(\mathscr{S}) A-A E(\mathscr{S})\|<\varepsilon$.

In the same vein, it is very easy to show that if $N$ is a normal operator and $\mathscr{K}$ is a subspace that reduces $N$, then $N$ restricted to $\mathscr{K}$ is also a normal operator. Again an asymptotic generalization of this fact may be conjectured as follows: If $N$ is a normal operator and $P$ is a projection onto a subspace $\mathscr{K}$ which "almost" reduces $N$, then the compression of $N$ to $\mathscr{K}$ is not far from the set of normal operators on $\mathscr{K}$; i.e., for every positive number $\varepsilon$, there exists a positive number $\delta$, such that $\|N P-P N\|<$ $\delta$ implies $\inf \left\{\left\|\left.P N P\right|_{\operatorname{ran}\left(I^{\prime}\right)}-N^{\prime}\right\|\right\}<\varepsilon$, where the infimum is taken over all normal operators $N^{\prime}$ on $\mathscr{K}$.

In this paper it is shown that both conjectures are true in finite-dimensional Hilbert space, and both are false in infinite-dimensional Hilbert space.

Received by the editors March 15, 1973.

AMS (MOS) subject classifications (1970). Primary 47B15, 47B20; Secondary 47A20, 47B05.

Key words and phrases. Normal, subnormal operators, spectral projections, normal approximation.

(c) American Mathematical Society 1974 


\section{Finite-dimensional spaces.}

THEOREM 1. If $N$ is a normal operator on a finite-dimensional Hilbert space, and if $\varepsilon>0$, then there exists a $\delta>0$ such that if $A$ is an operator satisfying $\|N A-A N\|<\delta$ then $\|A E(\mathscr{S})-E(\mathscr{S}) A\|<\varepsilon$, where $E$ is the unique spectral measure associated with $N$ and $\mathscr{S}$ is an arbitrary Borel subset of the plane.

Proof. Since there are only finitely many spectral projections, and since every spectral projection is a polynomial in the operator $N$, it is sufficient to show that if $\|N A-A N\|$ is small then $\left\|N^{t} A-A N^{t}\right\|$ is small for each $t$ in a finite set of positive integers. An easy calculation shows that $\left\|N^{t} A-A N^{t}\right\| \leqq t\|N\|^{l-1}\|N A-A N\|$, and the theorem is proved.

The following lemma is well known but has not appeared in print before. The proof is due to Halmos. A similar but nonequivalent statement was proved by W. A. J. Luxemburg and R. F. Taylor [2] using nonstandard analysis. The methods used in the proof below may be easily adapted to give the result of Luxemburg and Taylor.

Lemma 1. If $\mathscr{H}$ is a finite-dimensional Hilbert space, and if $\varepsilon>0$, then there exists a $\delta>0$ such that $\left\|A^{*} A-A A^{*}\right\|<\delta$ for any contraction $A$ implies there exists a normal operator $N$ satisfying $\|N-A\|<\varepsilon$.

Proof. Let $X$ be the unit ball in the (normed) space of operators on $\mathscr{H}$. For each $A$ in $X$ write $f(A)=\left\|A^{*} A-A A^{*}\right\|$. Then $f$ is a real-valued continuous function on a compact metric space. Let $\mathscr{N}=\{A \in X: f(A)=0\}$. Clearly $\mathcal{A}^{\circ}$ is the set of all normal operators in $X$. Let

$$
\mathscr{M}=\{A \in X:\|A-N\| \geqq \varepsilon \text { for all } N \text { in } \mathscr{N}\} \text {. }
$$

Then $\mathscr{M}$ is a compact subset of $X$ on which $f$ does not vanish. Let $\delta=$ $\inf \{f(A): A \in \mathscr{M}\}$. Then $\delta>0$ and if $f(A)<\delta$ for $A$ in $X$ then $A$ is not in $\mathscr{M}$. That is, there exists a normal operator $N$ such that $\|A-N\|<\varepsilon$.

A short description of the lemma is: "Almost" normal operators are "near" normal operators on a finite-dimensional Hilbert space.

THEOREM 2. If $N$ is a normal operator on a finite-dimensional Hilbert space $\mathscr{H}$, and if $\varepsilon>0$, then there exists a $\delta>0$ such that $\|N P-P N\|<\delta$ for some projection $P$ implies inf $\left\{\left\|\left.P N P\right|_{\operatorname{ran}}(1)-N^{\prime}\right\|\right\}<\varepsilon$ where the infimum is taken over all normal operators on $N^{\prime} \operatorname{ran}(P)$.

Proof. Let $P$ be any projection on $\mathscr{H}$, and let $\mathscr{K}$ denote $\operatorname{ran}(P)$. Then $N$ has a $2 \times 2$ operator matrix representation in the decomposition

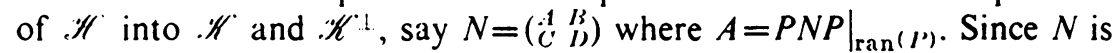
normal, it follows that $A^{*} A-A A^{*}=B B^{*}-C^{*} C$. Thus $\left\|A^{*} A-A A^{*}\right\| \leqq$ $\|B\|^{2}+\|C\|^{2}$. Since $P$ has the representation $\left(\begin{array}{ll}1 & 0 \\ 0 & 0\end{array}\right)$, it follows that 
$\|N P-P N\|=\sup \{\|B\|,\|C\|\}$. Hence, if $\|N P-P N\|$ is small, then $A$ is "almost" normal. By Lemma $1, A$ is "near" a normal and the proof is complete.

Infinite-dimensional spaces. In this section a normal operator is constructed on an infinite-dimensional Hilbert space which serves as a counterexample to both asymptotic generalizations. The primary tools are subnormal weighted shifts, the properties of their minimal normal extensions, and the following lemma which is due to Halmos.

Lemma 2. If $S$ is the unilateral shift on a separable Hilbert space, $N$ a normal operator, and $C$ a compact operator, then $\|S-(N+C)\| \geqq 1$.

Proof. Suppose there exists a normal operator $N$ and a compact operator $C$ such that $\|S-(N+C)\|<1$. Since $N$ can be approximated arbitrarily closely by an invertible normal operator, we may assume that $N$ is invertible. Since $\left\|I-S^{*}(N+C)\right\|<1$, it follows that $S^{*}(N+C)$ is invertible, and hence that $\operatorname{ker}(N+C)=0$. By an easy application of the Fredholm alternative, it follows that $N+C$ is invertible, and hence that $S^{*}$ is invertible, which is false. The proof of the lemma is complete.

A moment's glance at the proof reveals that the same statement is true for any nonunitary isometry. By consideration of the zero operator, the lemma implies that the distance from the unilateral shift to the set of operators of the form "normal plus compact" is equal to 1 .

THEOREM 3. There exist a normal operator $N$ with spectral measure $E$, a sequence of nonzero projections $\left\{P_{k}\right\}_{k=0}^{\infty}$, and a Borel subset $\mathscr{S}$ of the unit disk, with the following properties:

(1) $\operatorname{ran}\left(P_{k}\right)$ is invariant under $N$ for $k=0,1,2, \cdots$.

(2) $\left\|N P_{k}-P_{k} N\right\| \rightarrow 0$ as $k \rightarrow \infty$.

(3) $\inf \left\{\left\|\left.N\right|_{\operatorname{ran}\left(I_{k}^{\prime}\right)}-N^{\prime}\right\|\right\} \geqq 1$ where the infimum is taken over all normal operators $N^{\prime}$ on the $\operatorname{ran}\left(P_{k}\right), k=0,1,2, \cdots$.

(4) $\left\|E(\mathscr{S}) P_{k}-P_{k} E(\mathscr{S})\right\| \geqq 1 / 2 \pi$ for $k=0,1,2, \cdots$.

Proof. Consider the sequence $\left\{\mu_{k}\right\}_{k=0}^{\infty}$ of probability measures on the unit interval defined by

$$
d \mu_{k}(t)=(k+1)\left(1-t^{2}\right)^{k} 2 t \cdot d t .
$$

Let $\lambda$ denote normalized Lebesgue measure on the unit circle. Let $k$ be a fixed positive integer, and let $N_{k}$ be the position operator on $\mathscr{L}^{2}\left(\Delta, v_{k}\right)$, where $\Delta$ is the unit disk and $v_{k}$ is the Cartesian product measure $\mu_{k} \times \hat{\lambda}$ on the Borel subsets of the disk. For each positive integer $n$ let $f_{n}$ be function defined by $f_{n}(z)=z^{n}$, and let $e_{n}=f_{n} /\left\|f_{n}\right\|$. Let $\mathscr{H}^{2}\left(v_{k}\right)$ be the 
subspace spanned by the orthonormal set $\left\{e_{n}\right\}_{n=0}^{\infty}$. Let $A_{k}$ denote the restriction of $N_{k}$ to the invariant subspace $\mathscr{H}^{2}\left(v_{k}\right)$. Then, relative to the orthonormal basis $\left\{e_{n}\right\}_{n=0}^{\infty}, A_{k}$ is a weighted shift, and by a result of Berger $\left[1\right.$, pp. 895-897], its weight sequence $\left\{\alpha_{n}^{k}\right\}_{n=0}^{\infty}$ is defined by $\alpha_{n}^{k}=\rho_{n+1}^{k} / \rho_{n}^{k}$, where $\rho_{n}^{k}=\left(\int_{0}^{1} t^{2 n} d \mu_{k}(t)\right)^{1 / 2}$. It is easy to see that by the choice of the measure $\mu_{k}$,

$$
\alpha_{n}^{k}=((n+1) /(n+k+2))^{1 / 2} .
$$

Since $\lim _{n}\left\{\alpha_{n}^{k}\right\}=1$, it follows that $S-A_{k}$ is compact and $\left\|A_{k}\right\|=1$.

Let $Q_{k}$ be the projection of $\mathscr{L}^{2}\left(v_{k}\right)$ onto $\mathscr{H}^{2}\left(v_{k}\right)$. As previously noted, $\operatorname{ran}\left(Q_{k}\right)$ is invariant under $N_{k}$ (i). Then each $N_{k}$ has a $2 \times 2$ operator matrix representation in the decomposition of $\mathscr{L}^{2}\left(v_{k}\right)$ into $\mathscr{H}^{2}\left(v_{k}\right)$ and $\mathscr{H}^{2}\left(v_{k}\right)^{\perp}$, say

$$
N_{k}=\left(\begin{array}{ll}
A_{k} & B_{k} \\
0 & C_{k}
\end{array}\right) .
$$

Since $N_{k}$ is normal, it is easy to see that $\left\|B_{k}\right\|^{2}=\left\|A_{k}^{*} A_{k}-A_{k} A_{k}^{*}\right\|$. Since $Q_{k}$ has the $2 \times 2$ matrix representation $\left(\begin{array}{ll}1 & 0 \\ 0 & 0\end{array}\right)$, it follows that

$$
\left\|N_{k} Q_{k}-Q_{k} N_{k}\right\|^{2}=\left\|A_{k}^{*} A_{k}-A_{k} A_{k}^{*}\right\| .
$$

However, $A_{k}^{*} A_{k}-A_{k} A_{k}^{*}$ is a diagonal operator with eigenvectors the orthonormal basis $\left\{e_{n}\right\}_{n=0}^{\infty}$, and eigenvalues $\left\{\beta_{n}^{k}\right\}_{n=0}^{\infty}$, where

$$
\beta_{n}^{k}=(k+1) /(n+k+2)(n+k+1), \quad n=0,1,2, \cdots .
$$

Thus, $\left\|A_{k}^{*} A_{k}-A_{k} A_{k}^{*}\right\| \rightarrow 0$ as $k \rightarrow \infty$ and this implies that $\left\|N_{k} Q_{k}-Q_{k} N_{k}\right\| \rightarrow 0$ as $k \rightarrow \infty$ (ii). Since $S-A_{k}$ is compact and $\left.N_{k}\right|_{\operatorname{ran}\left(Q_{k}\right)}=A_{k}$, Lemma 2 implies that

$$
\left\|\left.N_{k}\right|_{\operatorname{ran}\left(Q_{k}\right)}-N^{\prime}\right\| \geqq 1
$$

for all normal operators $N^{\prime}$ on $\operatorname{ran}\left(Q_{k}\right)$ for each fixed positive integer $k$.

Let $\mathscr{S}=\left\{z=t e^{i \theta}: 0 \leqq t \leqq 1,-\pi / 4 \leqq \theta \leqq \pi / 4\right\}$, and let $E_{k}$ denote the spectral measure associated with the normal operator $N_{k}$. Let $f$ be the vector in $\mathscr{L}^{2}\left(v_{k}\right)$ defined by $f(z)=z$. It will be shown that

$$
\left\|\left(E_{k}(\mathscr{S}) Q_{k}-Q_{k} E_{k}(\mathscr{S})\right) f\right\| \geqq(1 / 2 \pi)\|f\| .
$$

This will establish that $\left\|E_{k}(\mathscr{S}) Q_{k}-Q_{k} E_{k}(\mathscr{S})\right\| \geqq 1 / 2 \pi$ (iv).

It is clear that $E_{k}(\mathscr{S})$ is multiplication by the characteristic function $\chi_{\mathscr{S}}$ of the set $\mathscr{S}$ on $\mathscr{L}^{2}\left(v_{k}\right)$. Since $Q_{k} f=f$, it follows that

$$
\left(E_{k}(\mathscr{S}) Q_{k}-Q_{k} E_{k}(\mathscr{S})\right) f=\left(1-Q_{k}\right)\left(\chi_{\mathscr{S}} \cdot f\right) .
$$


Since the vector $\bar{f}$ defined by $\bar{f}(z)=\bar{z}$ is orthogonal to $\operatorname{ran}\left(Q_{k}\right)$, the assertion will be established if it can be shown that

However,

$$
\left|\left(\bar{f},\left(\chi_{\mathscr{S}} \cdot f\right)\right)\right| \geqq(1 / 2 \pi)\|f\| \cdot\|\bar{f}\| .
$$

$$
\begin{aligned}
\left(\left(\chi_{\mathscr{S}} f\right), \bar{f}\right) & =\int_{\mathscr{S}} z^{2} d \nu_{k}(z)=\frac{1}{2 \pi}\left(\int_{-\pi / 4}^{\pi / 4} e^{2 i \theta} d \theta\right)\left(\int_{0}^{1} t^{2} d \mu_{k}(t)\right), \\
& =\frac{1}{2 \pi}\left(\int_{0}^{1} t^{2} d \mu_{k}(t)\right) .
\end{aligned}
$$

Since $\|f\|^{2}=\|\bar{f}\|^{2}=\int_{\Delta}|z|^{2} d v_{k}(z)=\int_{0}^{1} t^{2} d \mu_{k}(t)$, the assertion is proved.

Let $N$ be the infinite direct sum of the normal operators $\left\{N_{k}\right\}_{k=0}^{\infty}$, i.e., $N=\sum_{k=0}^{\infty} \oplus N_{k}$. Let $P_{k}$ be the projection defined by $P_{k}=\sum_{j=0}^{\infty} \oplus \delta_{j k} Q_{j}$, for $k=0,1,2, \cdots$. Then it follows that the spectral measure of $N$ is the direct sum of the spectral measures $E_{k}$ of the normal operators $N_{k}$. By (i) it follows that $\operatorname{ran}\left(P_{k}\right)\left(=\operatorname{ran}\left(Q_{k}\right)\right)$ is invariant under $N$ for $k=0,1,2, \cdots$. By (ii),

By (iii),

$$
\left\|N P_{k}-P_{k} N\right\|=\left\|N_{k} Q_{k}-Q_{k} N_{k}\right\| \rightarrow 0 \text { as } k \rightarrow \infty .
$$

$$
\left\|\left.N\right|_{\operatorname{ran}\left(P_{k}\right)}-N^{\prime}\right\|=\left\|\left.N_{k}\right|_{\operatorname{ran}\left(Q_{k}\right)}-N^{\prime}\right\| \geqq 1
$$

for all normal operators $N^{\prime}$ on the $\operatorname{ran}\left(P_{k}\right)$. By (iv),

$$
\left\|E(\mathscr{S}) P_{k}-P_{k} E(\mathscr{S})\right\|=\left\|E_{k}(\mathscr{S}) Q_{k}-Q_{k} E_{k}(\mathscr{S})\right\| \geqq 1 / 2 \pi .
$$

The theorem is proved.

\section{REFERENCES}

1. P. R. Halmos, Ten problems in Hilbert space, Bull. Amer. Math. Soc. 76 (1970), 887-933. MR 42 \#5066.

2. W. A. J. Luxemburg and R. F. Taylor, Almost commuting matrices are near commuting matrices, Nederl. Akad. Wetensch. Proc. Ser. A 73=Indag. Math. 32 (1970), 96-98. MR 41 \#3502.

Department of Mathematics, IndIANa UNIVERSITY, BlOOMINGTON, INDIANA 47401 (Current address of K. J. Harrison)

Current address (J. J. Bastian): Dalhousie University, Halifax, Nova Scotia, Canada 